BMJ Open

Diabetes

Research

\& Care

\title{
Objectively assessed physical activity, adiposity, and inflammatory markers in people with type 2 diabetes
}

\author{
Mark Hamer, Ruth A Hackett, Sophie Bostock, Antonio I Lazzarino, \\ Livia A Carvalho, Andrew Steptoe
}

To cite: Hamer M,

Hackett RA, Bostock S, et al. Objectively assessed physical activity, adiposity, and inflammatory markers in people with type 2 diabetes. BMJ Open Diabetes Research and Care 2014;2: 000030. doi:10.1136/bmjdrc-2014000030

- Additional material is available. To view please visit the journal (http://dx.doi.org/ 10.1136/bmjdrc-2014000030).

Received 12 March 2014 Revised 30 April 2014 Accepted 31 May 2014
CrossMark

Department of Epidemiology and Public Health, University College London, London, UK

Correspondence to Dr Mark Hamer; m.hamer@ucl.ac.uk

\section{ABSTRACT}

Objective: Inflammatory processes may play an important role in the development of acute coronary syndromes in people with type 2 diabetes; thus, strategies to control inflammation are of clinical importance. We examined the cross-sectional association between objectively assessed physical activity and inflammatory markers in a sample of people with type 2 diabetes.

Methods: Participants were 71 men and 41 women (mean age $=63.9 \pm 7$ years), without a history of cardiovascular disease, drawn from primary care clinics. Physical activity was objectively measured using waist-worn accelerometers (Actigraph GT3X) during waking hours for seven consecutive days.

Results: We observed inverse associations between moderate-to-vigorous physical activity ( per $10 \mathrm{~min}$ ) with plasma interleukin-6 $(\mathrm{B}=-0.035,95 \% \mathrm{Cl}-0.056$ to $-0.015)$, interleukin-1ra $(B=-0.033,95 \% \mathrm{Cl}-0.051$ to $-0.015)$, and monocyte chemotactic protein- $1(B=-0.011$, $95 \% \mathrm{Cl}-0.021$ to 0.000 ). These associations largely persisted in multivariable adjusted models, although body mass index considerably attenuated the effect estimate.

Conclusions: These data demonstrate an inverse association between physical activity and inflammatory markers in people with type 2 diabetes.

Inflammatory processes are believed to play an important role in the prognosis of people with type 2 diabetes, for example, being linked with the risk of end-organ damage, such as diabetic retinopathy and cardiovascular disease (CVD). ${ }^{12}$ Inflammation plays a pivotal role in vulnerable plaque formation through lesion necrosis and thinning of the fibrous capsule that can result in endothelial erosion or plaque rupture, leading to acute coronary syndromes. ${ }^{3}$ Thus, strategies to control low-grade inflammation are of clinical importance.

Evidence from epidemiological studies of healthy populations has demonstrated an inverse association between physical activity (PA) and markers of low-grade systemic inflammation. $^{4-6}$ Until now, most studies have concentrated on $\mathrm{C}$ reactive protein (CRP) due to the development of low-cost reliable assays and commonplace use in

\section{Key messages}

Physical activity is beneficial for controlling diabetes, but the mechanisms remain poorly understood.

- In this study, objectively assessed moderate-to-vigorous intensity physical activity was associated with novel inflammatory markers.

- The inverse association between physical activity and inflammatory markers was partly explained through adiposity.

clinical practice. Other markers of inflammation, such as plasma interleukin-6 (IL-6) and monocyte chemotactic protein-1 (MCP-1), may be more relevant in the context of exercise. The anti-inflammatory effects of exercise could be explained by adiposity and non-adiposity-related mechanisms. Exercise could decrease the migration of macrophages to adipocytes via reduced proinflammatory cytokines such as IL-6 and MCP-1, or decrease adipose tissue macrophages in the production of IL-6 and MCP-1. ${ }^{78}$

Data are lacking in people with diabetes, particularly using objective assessments of PA. Objective assessment of PA is being increasingly used to overcome biases of selfreport and provide data on the entire intensity spectrum of PA across waking hours. ${ }^{9}$ The aim of this study was to examine the association between objectively assessed PA, adiposity, and novel inflammatory markers in a sample of people with type 2 diabetes.

\section{RESEARCH DESIGN AND MIETHODS}

People with type 2 diabetes but without a history of CVD were recruited from primary care clinics in London and the University College London hospital outpatient clinic. We approached 36 clinics in the North London area providing information sheets about the study. General practitioners from 18 clinics agreed to participate and subsequently 
recruited eligible patients. History of CVD was defined by physician diagnosis, which was verified by the general practitioner when recruiting patients into the study. Participants gave full informed written consent to participate in the study. Based on our previous research, ${ }^{5}{ }^{6}$ we anticipated low-moderate effect sizes $(\mathrm{d}=0.25)$. Thus, a sample size of $\mathrm{N}=100$ would provide us with $80 \%$ power at $5 \%$ significance level (two-tailed) to detect differences in inflammatory markers relative to PA.

Participants wore an accelerometer (Actigraph GT3X, Florida, USA) around the waist that records movement on the vertical and horizontal axis, during waking hours for seven consecutive days. The accelerometer provides a measure of the frequency, intensity, and duration of PA and allows classification of activity levels as sedentary, light, moderate, and vigorous. The raw accelerometry data were processed in $60 \mathrm{~s}$ epochs using specialist software (MAHUffe, Cambridge, UK) to produce a series of standardized variables. All participants included in the present analysis recorded a minimum of $10 \mathrm{~h} /$ day wear time and provided data for 5 days after exclusion of the first and last days of wear. Non-wear time was defined as intervals of at least 60 consecutive minutes of $0 \mathrm{cpm}$. We used cutoff points previously employed in samples of older British adults ${ }^{10}$ to calculate daily times in each activity intensity band: sedentary $(<1.5$ MET $)$ 0-199 cpm, light (1.5-3 MET) 200-1998 cpm,

Table 1 Descriptive characteristics $(n=112)$

\begin{tabular}{ll}
\hline Variable* $^{*}$ & \\
\hline Age & $63.9 \pm 7.0$ \\
Sex (\% men) & $71(63.4)$ \\
Smokers (\%) & $16(14.4)$ \\
Ethnicity (\%) & \\
$\quad$ White & $91(81.3)$ \\
$\quad$ Asian & $9(8.0)$ \\
Afro-Caribbean & $6(5.4)$ \\
Other & $6(5.4)$ \\
Sedentary (min/day) & $659.6 \pm 111.6$ \\
Light activity (min/day) & $186.9 \pm 70.7$ \\
MVPA (min/day) & $32.1 \pm 23.2$ \\
Actigraph wear time (min/day) & $878.5 \pm 102.2$ \\
Body mass index (kg/m²) & $30.9 \pm 5.6$ \\
HbA1c (\% and mmol/mol) & $7.2 \pm 1.3$ \\
& $(55 \pm 14.2)$ \\
Interleukin-6 (pg/mL) & $2.1 \pm 1.3$ \\
Interleukin-1ra (pg/mL) & $897.1 \pm 571.8$ \\
Monocyte chemotactic protein-1 (pg/mL) & $119.3 \pm 32.7$ \\
Medications (\%) & \\
Oral antidiabetic & $92(82.4)$ \\
Injectable antidiabetic and insulin & $11(10.2)$ \\
Antihypertensive & $80(71.3)$ \\
Lipid lowering & $87(77.8)$ \\
Aspirin & $41(36.2)$ \\
\hline *Data are presented as mean $\pm S D$ unless otherwise stated. \\
Percentages are denoted in brackets. \\
HbA1c, glycated hemoglobin; MVPA, moderate-to-vigorous \\
physical activity. \\
\end{tabular}

moderate-to-vigorous PA (MVPA; >3 MET) $\geq 1999 \mathrm{cpm}$. All PA variables were converted to time (minutes) in specific PA intensities per valid day.

Following the week of Actigraph assessment, participants reported to the laboratory for clinical assessments. Measurements included height, weight, waist and hip circumference, blood pressure, and a non-fasting blood sample taken from the antecubital fossa. BMI was calculated using weight $(\mathrm{kg}) /\left(\right.$ height $\left.\left(\mathrm{m}^{2}\right)\right)$. Glycated hemoglobin (HbAlc) concentration was assayed using boronate affinity chromatography, a combination of boronate affinity and liquid chromatography. The inflammatory marker IL-6 was measured using a high-sensitivity ELISA (R\&D Systems, Oxford, UK). Other inflammatory markers included IL-1ra and MCP-1, which were assayed using the Magnetic Luminex Performance Assay Human CCL2/ MCP-1 multiplexed IL-1RA kit (R\&D Systems) read on a Luminex 200 (Bio-Rad). Coefficients of variation for all assays were less than $8 \%$.

The primary outcome was IL- 6 and analyses with other more novel inflammatory markers (IL-1ra, MCP-1) were treated as exploratory. Multiple linear regression was employed to examine the cross-sectional association between MVPA and log-transformed inflammatory markers, making adjustments for Actigraph wear time, age, sex, smoking, ethnicity, medications, HbAlc, and finally BMI. We performed additional analyses to examine associations between sedentary time and inflammatory markers using the same statistical approach. All analyses were conducted using SPSS V.21.

\section{RESULTS}

We recruited 140 participants, although after exclusion of missing data ( $\mathrm{N}=16$ missing Actigraph data due to technical failure; $\mathrm{N}=12$ missing bloods) our analytic sample comprised 112 men and women. Excluded participants did not differ from the analytic sample on any variables $(\mathrm{eg}$, age $=63.1 \pm 7$ vs $63.9 \pm 7, \mathrm{p}=0.63)$. The descriptive characteristics of the sample are displayed in table 1. Participants ranged from 50 to 75 years of age, were largely Caucasian, and $48.2 \%$ were obese. The majority $(82.4 \%)$ of the sample was prescribed metformin, and in $34.8 \%$ of them HbAlc was less than $6.5 \%$ $(48 \mathrm{mmol} / \mathrm{mol})$; in $33.9 \%$ it was between $6.5 \%$ and $7.5 \%(48-58 \mathrm{mmol} / \mathrm{mol})$, and in $31.3 \%$ it was over $7.5 \%$ $(58 \mathrm{mmol} / \mathrm{mol})$. Participants wore the Actigraph on average for $14.6 \mathrm{~h} /$ day, and $75.1 \%, 21.3 \%$, and $3.6 \%$ of wear time was spent in sedentary, light, and moderate-to-vigorous activity categories, respectively.

MVPA was inversely associated with HbAlc (per 10 min increase, $\mathrm{B}=-0.13,95 \% \mathrm{CI}-0.25$ to -0.06 ), and with $\mathrm{BMI}$ (per 10 min increase, $\mathrm{B}=-0.80,95 \% \mathrm{CI}-1.23$ to -0.37) after adjustment for covariables. We also observed inverse associations between MVPA and all inflammatory markers (table 2). The associations for IL-6 and IL-1ra persisted in multivariable adjusted models, although BMI considerably attenuated the 
Table 2 Regression of MVPA on inflammatory markers

\begin{tabular}{llll}
\hline & Model 1 & Model 2 & Model 3 \\
& B (95\% Cl) & B (95\% Cl) & B (95\% Cl) \\
\hline Interleukin-6 & $-0.035(-0.056$ to -0.015$)$ & $-0.035(-0.056$ to -0.014$)$ & $-0.022(-0.043$ to -0.001$)$ \\
Interleukin-1ra & $-0.033(-0.051$ to -0.015$)$ & $-0.032(-0.050$ to -0.013$)$ & $-0.014(-0.030$ to 0.003$)$ \\
Monocyte chemotactic protein-1 & $-0.011(-0.021$ to 0.000$)$ & $-0.009(-0.020$ to 0.002$)$ & $-0.007(-0.019$ to 0.005$)$ \\
\hline Coefficients (unstandardized B) represent a 10 min increase in MVPA. & & \\
Model 1: adjusted for age, sex, Actigraph wear time. & & \\
Model 2: additionally adjusted for smoking, ethnicity, HbA1c, total number of medications. \\
Model 3: additionally adjusted for BMl. \\
BMI, body mass index; HbA1c, glycated hemoglobin; MVPA, moderate-to-vigorous physical activity.
\end{tabular}

effects. Indeed, BMI was directly associated with IL-6 and IL-1ra, although not with MCP-1 (see online supplementary table S1). In further sensitivity analyses, we adjusted for individual types of medication with antiinflammatory properties (instead of total number), including statins, $\beta$-blockers, aspirin, and metformin. The results, however, remained unchanged in all analyses.

We also examined associations between other domains of activity, including sedentary and light activity, with inflammatory markers. There was an association between sedentary time and IL-6 (per 10 min increase, $\mathrm{B}=0.007,95 \%$ CI 0.00 to $0.013, \mathrm{p}=0.053$ ) after adjustment for MVPA and all other covariables, although not with any of the other inflammatory markers. There were no associations between light-intensity activity and any inflammatory markers (data not shown).

\section{DISCUSSION}

PA may be an important intervention to reduce inflammatory markers in clinical groups, ${ }^{11}$ although there are currently little objective data in people with type 2 diabetes. We observed inverse associations between objectively assessed PA and several markers of inflammation. In particular, a novel finding was the association with MCP-1. MCP-1 secreted from white adipose tissue is linked to tissue macrophage accumulation and insulin resistance by inducing a chronic inflammatory state. ${ }^{12}$ Our data are consistent with several recent studies that observed inverse, dose-response associations between objectively assessed PA and inflammatory markers among people with type 2 diabetes. ${ }^{13} 14$

The anti-inflammatory effects of exercise could be explained by adiposity and non-adiposity related mechanisms. Exercise could decrease the migration of macrophages to adipocytes via reduced proinflammatory cytokines such as IL-6 and MCP-1, or decrease adipose tissue macrophages in the production of IL- 6 and MCP-1. ${ }^{7}$ The expression of exercise-regulated muscle genes, such as the transcriptional coactivator PGC1 $\alpha$, is thought to promote anti-inflammatory effects through a transient release of cytokines, and possibly explains some of the systemic and beneficial effects of exercise in non-muscle tissue. ${ }^{8}$ Epidemiological and clinical trials also suggest that adiposity and non-adiposity related mechanisms play a role in the anti-inflammatory effect of exercise. Our data suggest that BMI largely explained the associations between PA and inflammatory markers. However, in previous epidemiological studies of healthy participants, the association between PA and markers of low-grade systemic inflammation is largely independent of adiposity. ${ }^{15}$ Data from clinical trials are also inconsistent. In the 1-year follow-up of the Finnish Diabetes Prevention study, increased moderate-to-vigorous activity was associated with a reduction in CRP and IL-6, independently of baseline BMI or changes in BMI. ${ }^{16}$ In a 10 -month randomized trial of older adults, aerobic exercise training resulted in reduced CRP, and these effects were partly mediated through a reduction in central adiposity. ${ }^{17}$ Similarly, in a year-long trial of moderate exercise training, CRP was lowered only in women who were obese at study entry and only in participants who lost greater than $2 \%$ body fat. ${ }^{18}$ These inconsistencies may, in part, be also explained by the use of a variety of different inflammatory markers, some of which may act via separate mechanistic pathways. Nevertheless, the role of weight loss remains unclear, as a recent intensive lifestyle intervention focusing on weight loss did not reduce the rate of cardiovascular events in overweight or obese adults with type 2 diabetes. ${ }^{19}$ This trial has, however, received criticism including the use of more cardioprotective medicines in the control group and lack of statistical power for a longer duration of intensive lifestyle changes.

Inconsistencies in the literature might also be explained by the use of crude assessments of adiposity, as more precise measures of regional body fat tend to reveal stronger mediating effects. ${ }^{17}$ In the present study, BMI was strongly associated with IL-6 and IL-1ra, but not with MCP-1. In previous studies, MCP-1 was associated with visceral but not subcutaneous adipose tissue. ${ }^{20}$ Such subtle effects are difficult to detect using only BMI.

There has been substantial interest in the association between sedentary activity and cardiometabolic health. $^{21} 22$ In our study, we have found inconsistent associations between sedentary activity and inflammatory markers, which are in contrast with a previous work in healthy participants where associations between objective sedentary time and CRP/IL-6 were observed after controlling for MVPA. ${ }^{21} 22$ In our study, however, the most consistent associations were observed for MVPA, 
suggesting that participation in only moderate-to-intense exercise decreases inflammatory markers in people with type 2 diabetes. Further randomized trials are, however, required to confirm this. The count-based approach used to assess PA intensity thresholds in this study has some limitations, particularly in distinguishing between sedentary and light activities. In sensitivity analyses, however, using different cut points did not change the results (data not shown).

The main limitation of this study is the cross-sectional design, which precludes us from making any inferences about direction or causality. However, a key strength was the selection of community-dwelling people with diabetes from primary care and outpatient clinics. None of the participants had a history of CVD and the analysis was adjusted for markers of diabetes control (HbAlc) and medications as a marker of disease burden. Thus, although reverse causation is possible, in that individuals with more severe diabetes and comorbidity were less able to undertake PA, it is unlikely to explain our results. A further key strength was the use of objective PA measures, which eliminated problems of recall bias that plague more traditional self-report methods.

In summary, we have observed inverse associations between objectively assessed PA and several inflammatory markers. These data further support the beneficial effects of moderate-intensity PA in the management of diabetes.

Contributors MH had full access to the data, and takes responsibility for the integrity and accuracy of the results. MH drafted the paper. All authors contributed to the concept and design of the study, drafting and critical revision of the manuscript. All authors have approved the final version to be published.

Funding The British Heart Foundation (RE/10/005/28296).

Competing interests None.

Patient consent Obtained.

Ethics approval University College London Hospital committee on the Ethics of Human Research.

Provenance and peer review Not commissioned; externally peer reviewed.

Data sharing statement No additional data are available.

Open Access This is an Open Access article distributed in accordance with the Creative Commons Attribution Non Commercial (CC BY-NC 3.0) license, which permits others to distribute, remix, adapt, build upon this work noncommercially, and license their derivative works on different terms, provided the original work is properly cited and the use is non-commercial. See: http:// creativecommons.org/licenses/by-nc/3.0/

\section{REFERENCES}

1. Antonetti DA, Klein R, Gardner TW. Diabetic retinopathy. N Engl J Med 2012;366:1227-39.
2. Soinio M, Marniemi J, Laakso M, et al. High sensitivity C-reactive protein and coronary heart disease mortality in patients with type 2 diabetes: a 7-year follow-up study. Diabetes Care 2006;29:329-33.

3. Libby P. Mechanisms of acute coronary syndromes and their implications for therapy. N Engl J Med 2013;368:2004-13.

4. Gleeson M, Bishop NC, Stensel DJ, et al. The anti-inflammatory effects of exercise: mechanisms and implications for the prevention and treatment of disease. Nat Rev Immunol 2011;11:607-15.

5. Hamer M, Sabia S, Batty GD, et al. Physical activity and inflammatory markers over 10 years: follow-up in men and women from the Whitehall II cohort study. Circulation 2012;126:928-33.

6. Hamer M, Steptoe A. Prospective study of physical fitness, adiposity and inflammatory markers in healthy middle aged men and women. Am J Clin Nutr 2009;89:85-9.

7. Kanda H, Tateya S, Tamori Y, et al. MCP-1 contributes to macrophage infiltration into adipose tissue, insulin resistance, and hepatic steatosis in obesity. J Clin Invest 2006:116:1494-505.

8. Handschin C, Spiegelman BM. The role of exercise and PGC1alpha in inflammation and chronic disease. Nature 2008;454:463-9.

9. Strath SJ, Kaminsky LA, Ainsworth BE, et al. American Heart Association Physical Activity Committee of the Council on Lifestyle and Cardiometabolic Health and Cardiovascular, Exercise, Cardiac Rehabilitation and Prevention Committee of the Council on Clinical Cardiology, and Council. Guide to the assessment of physical activity: clinical and research applications: a scientific statement from the American heart association. Circulation 2013;128:2259-79.

10. Harris TJ, Owen CG, Victor CR, et al. What factors are associated with physical activity in older people, assessed objectively by accelerometry? Br J Sports Med 2009;43:442-50.

11. Ploeger HE, Takken T, de Greef MH, et al. The effects of acute and chronic exercise on inflammatory markers in children and adults with a chronic inflammatory disease: a systematic review. Exerc Immunol Rev 2009;15:6-41.

12. Nio Y, Yamauchi T, Iwabu M, et al. Monocyte chemoattractant protein-1 (MCP-1) deficiency enhances alternatively activated M2 macrophages and ameliorates insulin resistance and fatty liver in lipoatrophic diabetic A-ZIP transgenic mice. Diabetologia 2012;55:3350-8.

13. Loprinzi PD, Ramulu PY. Objectively measured physical activity and inflammatory markers among US adults with diabetes: implications for attenuating disease progression. Mayo Clin Proc 2013;88:942-51.

14. Jennersjö P, Ludvigsson J, Länne $\mathrm{T}$, et al. Pedometer-determined physical activity is linked to low systemic inflammation and low arterial stiffness in type 2 diabetes. Diabet Med 2012;29:1119-25.

15. Hamer $M$. The relative influence of fitness and fatness on inflammatory factors. Prev Med 2007:44:3-11.

16. Herder $\mathrm{C}$, Peltonen M, Koenig W, et al. Finnish Diabetes Prevention Study Group. Anti-inflammatory effect of lifestyle changes in the Finnish Diabetes Prevention Study. Diabetologia 2009;52:433-42.

17. Vieira VJ, Hu L, Valentine RJ, et al. Reduction in trunk fat predicts cardiovascular exercise training-related reductions in C-reactive protein. Brain Behav Immun 2009;23:485-91.

18. Campbell PT, Campbell KL, Wener MH, et al. A yearlong exercise intervention decreases CRP among obese postmenopausal women. Med Sci Sports Exerc 2009;41:1533-9.

19. Wing RR, Bolin P, Brancati FL, et al. Look AHEAD Research Group. Cardiovascular effects of intensive lifestyle intervention in type 2 diabetes. N Engl J Med 2013;369:145-54.

20. Pou KM, Massaro JM, Hoffmann U, et al. Visceral and subcutaneous adipose tissue volumes are cross-sectionally related to markers of inflammation and oxidative stress: the Framingham Heart Study. Circulation 2007;116:1234-41.

21. Healy GN, Matthews CE, Dunstan DW, et al. Sedentary time and cardio-metabolic biomarkers in US adults: NHANES 2003-06. Eur Heart J 2011;32:590-7.

22. Henson J, Yates T, Edwardson CL, et al. Sedentary time and markers of chronic low-grade inflammation in a high risk population. PLOS ONE 2013;8:e78350. 